\title{
Control Techniques of the Auxiliary-Resonant Commutated Pole With Special Regards on the Dual-Active Bridge DC-DC Converter
}

\author{
Johannes Voss, Jochen Henn, and Rik W. De Doncker
}

\begin{abstract}
In this work, a dual-active bridge dc-dc converter with auxiliary-resonant commutated pole is presented. The paper focuses on the control strategies of the auxiliary-resonant commutated pole to allow soft turn on and soft turn off of the main switches, and thus increasing the efficiency of the converter. These strategies are developed for a high-power IGCT-based dual-active bridge dc-dc converter. Two boosting strategies, constant time magnetization and constant current boosting are presented and tested. Besides its main objective to reduce the switching losses, the ARCP also allows balancing of the dc-link voltages in case of imbalanced loads. Strategies for boost current control and active dc-link balancing are introduced, presented and experimentally verified on a small-scale laboratory setup.
\end{abstract}

Index terms-DC-DC power converters, field programmable gate arrays, resonant inverters, soft-switching, zero-voltage switching.

\section{INTRODUCTION AND MotivATION}

$\mathrm{I}^{\mathrm{N}}$ Fig. 1, the schematic of a dual-active bridge (DAB) dc-dc converter with auxiliary-resonant commutated pole (ARCP) is shown. In case one of the main devices is switched, the ARCP causes a resonance to allow a zero-voltage condition at the main devices which then results in soft switching. This soft-switching behavior can be supported by additional resonant capacitors $C_{\text {res. }}$ In case of using ARCP with IGCTs, it has been shown that the switching losses can be reduced to an eighth of the original amount [7]. Further details on the DAB can be found in [1] and [8] and on the ARCP in [2] and [9]. The presented control techniques for the ARCP are developed for a $5 \mathrm{MW}$ DAB. Therefore, some presented hardware components are designed for $5 \mathrm{kV}$ and fiber optical logic signals are used. The techniques are tested on a smallscale model (SS-DAB) rated for powers in the lower $\mathrm{kW}$ range to minimize risks.

\section{A. Auxiliary-Resonant Commutated-Pole for Dual-Active Bridge}

As described in [1], a two-level three-phase DAB operates

Manuscript received May 22, 2018. This work is part of the research conducted in the Forschungscampus Flexible Electrical Networks Project 2: Equipment and Network Technologies for Medium-Voltage DC Applications, funded by the German Federal Ministry of Education and Research (03SF0489).

The authors are with the Institute for Power Generation and Storage Systems, E.On Energy Research Center, RWTH Aachen University, Aachen, Germany (e-mail: post_pgs@eonerc.rwth-aachen.de).

Digital Object Identifier 10.24295/CPSSTPEA.2018.00034 in six-step mode where each bridge is switched with a constant duty cycle of $50 \%$. The three phases of the bridge are shifted by $120^{\circ}$ generating a six-step voltage waveform at the transformer terminal. The phase shift between the primary and secondary bridges causes a voltage drop across the transformer leakage inductance, resulting in a power flow.

In partial load conditions or unsymmetrical input/output voltages, the $\mathrm{DAB}$ converter loses its soft-switching capability because there is not enough reactive current to discharge the snubber capacitors of the turning off device. Hence, the turning off devices are hard switched. The ARCP circuit is a promising technique to overcome this problem, thus extending the softswitching capability of the DAB. The main principle is to force a resonance just before a commutation process from a bottom switch to a top switch (BtT) or vice versa (TtB) so that the snubber capacitors in parallel to the switched device are fully discharged and charged, respectively [2], [4]. The main switch is then triggered under a zero-voltage condition. Especially thyristor-based turn-off devices (GTOs or IGCTs) have a deeper charge carrier saturation [3] and, therefore, introduce higher switching losses as compared to their competitive devices, e.g. Insulate-Gate Bipolar Transistors (IGBTs). To overcome the drawback of high switching losses and to take advantage of the superior conduction behavior of IGCTs, an ARCP can be applied to the DAB.

\section{B. Control Techniques Supporting Soft-Switching}

In Fig. 4, one phase-leg of the three-phase DAB with ARCP (DAB ARCP) is shown. A more detailed description of the basic ARCP principle can be found in [2] and [5]. The auxiliary inductor $L_{\mathrm{AUX}}$ needs to be tuned, according to the $\mathrm{d} i / \mathrm{d} t$ characteristics of the device in the converter with the lowest $\mathrm{d} i / \mathrm{d} t$ capability. In case the converter legs consist of IGBTs, no restriction for $\mathrm{d} i / \mathrm{d} t$ exists. However, IGCTs have $\mathrm{d} i / \mathrm{d} t$ limitations (e.g. $800 \mathrm{~A} / \mu \mathrm{s})$ which make a conservative design of $L_{\mathrm{AUX}}$ necessary.

Furthermore, Fig. 4 introduces a zero-voltage detection (ZVD). This ZVD is needed to switch on the main devices during the zero-voltage condition and to protect them from short-circuiting the resonant capacitors $C_{\text {res }}$ in case they are not completely discharged. Since the resonance phase is in the range of 10-30 $\mu$ s the ZVD must be very fast to avoid a flip back of the voltages across the resonant capacitors. A controller unit triggers the switch via the ZVD: whenever the ZVD 


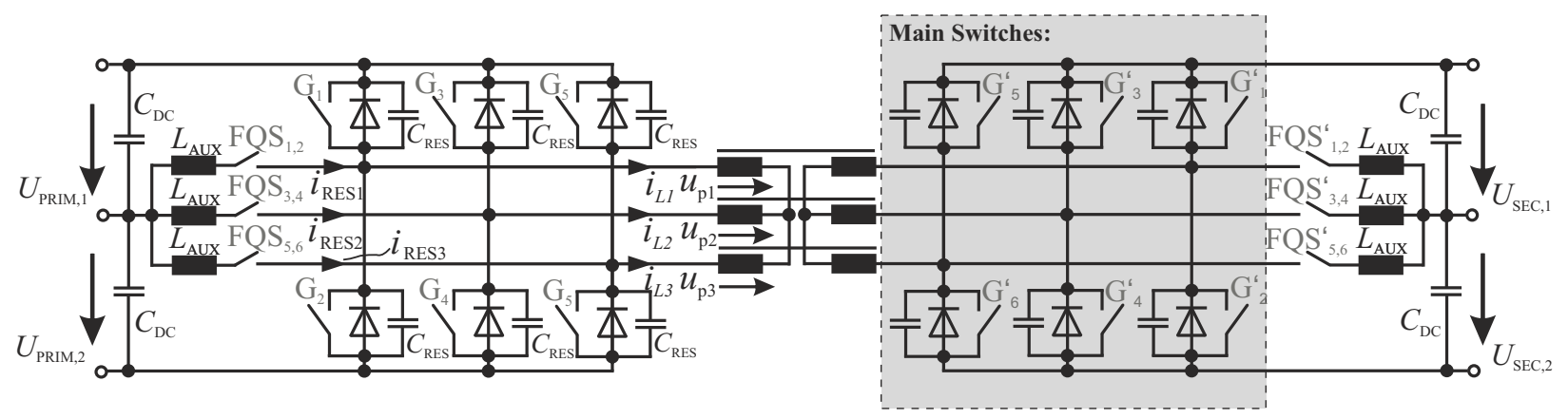

Fig. 1. Dual-active bridge with auxiliary-resonant commutated pole.

TABLE I

Space Vectors (SV) and Thyristor Triggering Signals FQS for One ConVerter

\begin{tabular}{cccc}
\hline \hline Time Sequence & SV & FQS & State \\
\hline$-T_{\text {discharge }}<t<0$ & 000000 & 000000 & 0 \\
$-T_{\mathrm{trg}}<t<0$ & 010101 & 010000 & $0-1$ \\
$0<t<T_{\mathrm{sw}} / 6$ & 100101 & 000000 & 1 \\
$T_{\mathrm{sw}} / 6-T_{\mathrm{trg}}<t<T_{\mathrm{sw}} / 6$ & 100101 & 000100 & $1-2$ \\
$T_{\mathrm{sw}} / 6<t<2 T_{\mathrm{sw}} / 6$ & 101001 & 000000 & 2 \\
$2 T_{\mathrm{sw}} / 6-T_{\mathrm{trg}}<t<2 T_{\mathrm{sw}} / 6$ & 101001 & 100000 & $2-3$ \\
$2 T_{\mathrm{sw}} / 6<t<3 T_{\mathrm{sw}} / 6$ & 011001 & 000000 & 3 \\
$3 T_{\mathrm{sw}} / 6-T_{\mathrm{trg}}<t<3 T_{\mathrm{sw}} / 6$ & 011001 & 000001 & $3-4$ \\
$3 T_{\mathrm{sw}} / 6<t<4 T_{\mathrm{sw}} / 6$ & 011010 & 000000 & 4 \\
$4 T_{\mathrm{sw}} / 6-T_{\mathrm{trg}}<t<4 T_{\mathrm{sw}} / 6$ & 011010 & 001000 & $4-5$ \\
$4 T_{\mathrm{sw}} / 6<t<5 T_{\mathrm{sw}} / 6$ & 010110 & 000000 & 5 \\
$5 T_{\mathrm{sw}} / 6-T_{\mathrm{trg}}<t<5 T_{\mathrm{sw}} / 6$ & 010110 & 010000 & $5-6$ \\
$5 T_{\mathrm{sw}} / 6<t<6 T_{\mathrm{sw}} / 6$ & 100110 & 000000 & 6 \\
$6 T_{\mathrm{sw}} / 6-T_{\mathrm{trg}}<t<6 T_{\mathrm{sw}} / 6$ & 100110 & 000010 & $6-1$ \\
\hline \hline
\end{tabular}

detects a turn-on signal and a zero-voltage condition at the main device, it is triggered on (logic AND). In case the controller unit generates a trigger signal without having zero-voltage condition, the ZVD does not trigger the main devices and thus avoiding a snubber dump.

As given in literature [8], [2] and [11], a boost current is an additional current which is needed to overcome losses during switching and to force a current into the anti-parallel diodes.

In this paper, two boosting strategies are introduced: the constant boost time and the constant boost current. By iterative simulation and loss estimation, the minimal boost time can be determined for various but steady operating points of the converter, as it has been shown in [10]. Instead of setting a constant boost time, a constant boost current can be obtained by using additional hardware. Since in dc grids a voltage variation of $+/-10 \%$ may exist, a constant boost time will lead to different boosting currents, i.e. an increased dc-link voltage causes a linear increasing ARCP peak current. By choosing a constant boost current technique, it is guaranteed to have the same boost

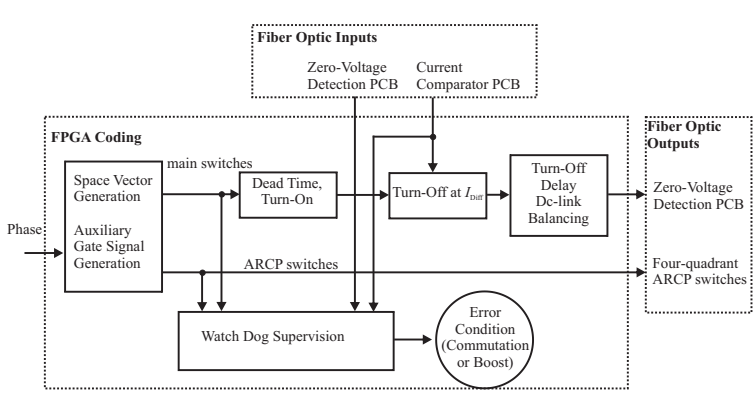

Fig. 2. Structure of the control algorithms in the FPGA.

current in every operation point of the converter.

\section{Control of the DAB With ARCP}

In this section the generation of gate signals is explained. The code has been verified in Matlab/Simulink using the Xilinx System Generator. In TABLE I, the switching sequence for the space vectors (SV) controlling the main converter legs and the four-quadrant switches (FQS) for the auxiliary branch are programmed on the FPGA ${ }^{1}$. Since thyristors are linecommutated devices, ' 0 ' does not necessarily mean that the device is not conducting current. Whenever the thyristors is triggered it carries current until it decays to zero. During $T_{\text {discharge }}$ the resonant capacitor has to be discharged by the zerostate chopper (ZSC). Without using a ZSC, the converter is not able to start from an initial space vector ' 000 ' as given in the TABLE I with state 0 , since the snubber capacitors of the device would share the dc-link voltage equally and the ZVD will not allow to trigger the main devices. Hard-switching with IGBTs might not be a critical, due to their high over current capability without $\mathrm{d} i / \mathrm{d} t$ limitation. In case of IGCTs this is crucial aspect. $T_{\mathrm{sw}}$ defines the switching period and $T_{\text {trg }}$ marks the magnetization time of the resonant inductor. Whenever a state should be changed, the corresponding FQS is triggered to realize the resonant commutation of a leg (e.g. from state 0 to state 1 only $\mathrm{FQS}_{1}$ must be triggered). In total, 12 gate signals for the main switches and 12 FQS signals are generated for the primary and secondary side. As shown in Fig. 2 a turn-on delay is embedded to prevent a short circuit on the dc link in any case. The turnoff block given in Fig. 2 is only applied when constant boost

\footnotetext{
' ' 1 ' means a switch is triggered on, and ' 0 ' a switch is not triggered, e.g. $\mathrm{SV}\left(\mathrm{G}_{1}, \mathrm{G}_{2}, \mathrm{G}_{3}, \mathrm{G}_{4}, \mathrm{G}_{5}, \mathrm{G}_{6}\right)=$ '000000' means all switches are not triggered.
} 


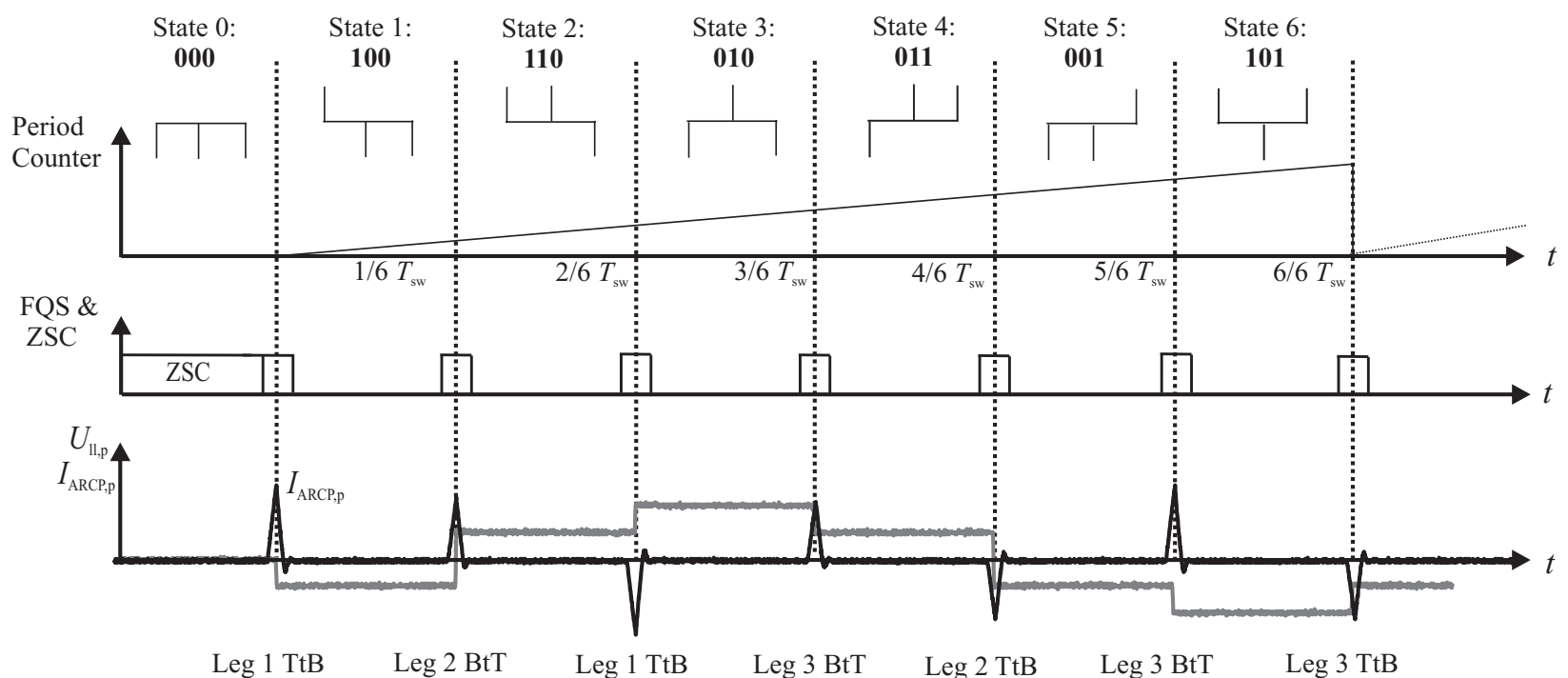

Fig. 3. Space vectors and the resulting phase voltage of the $3 \mathrm{PH} \mathrm{DAB}$ including the ARCP currents.
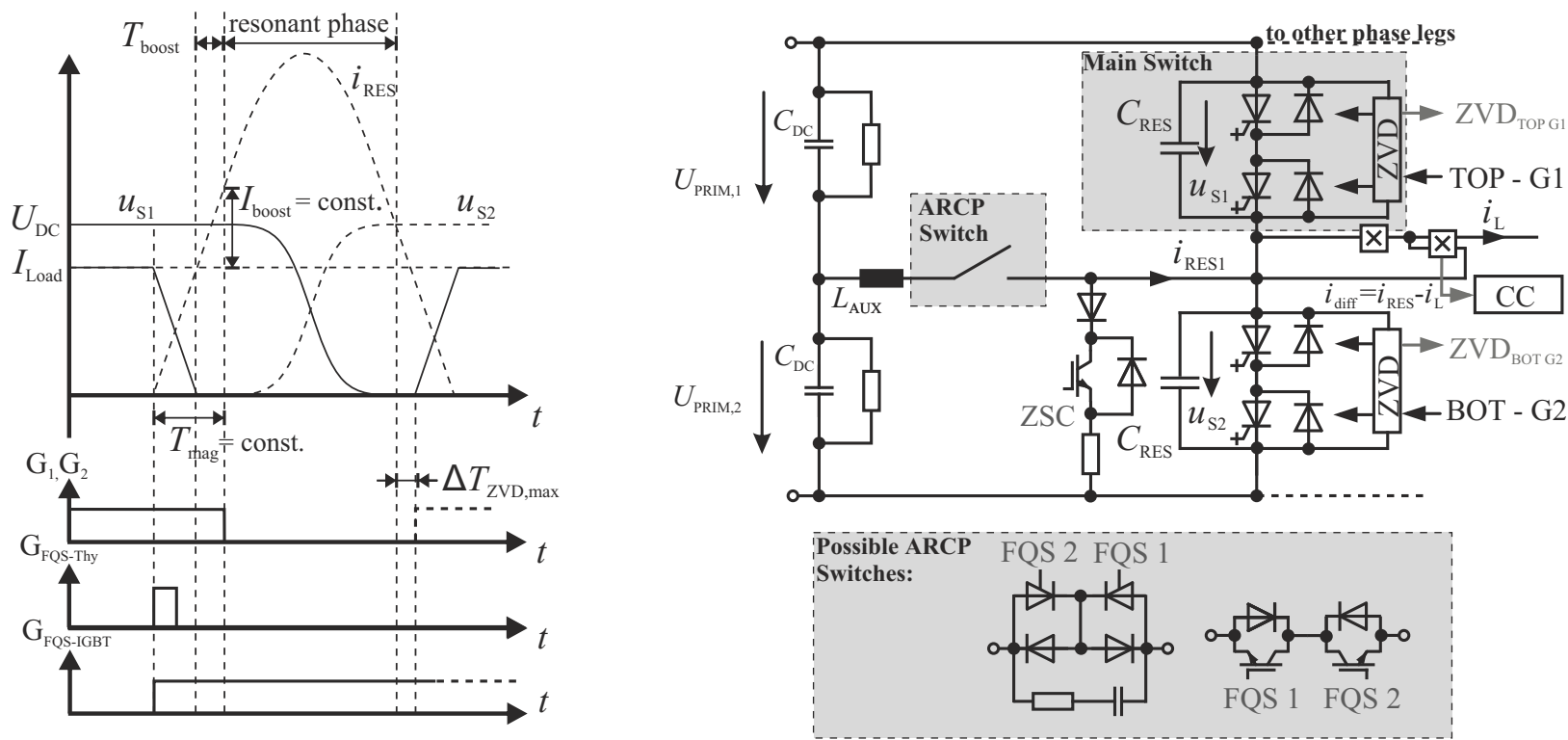

Fig. 4. Boosting strategies for ARCP current: Either constant magnetization time or constant boost current using comparator PCB.

current mode is applied, which will be explained in the next section.

In TABLE I and TABLE II, the basic timings for the main switches and the FQS are given. Various topologies of semiconductor for the four-quadrant switch (FQS) are possible. Since the resonant phase ends at a zero-current crossing linecommutated and forced-commutated devices can be utilized. In case of line-commutated devices, namely thyristors, only a short trigger impulse has to be applied to start the resonant phase. If forced-commutated devices are used (e.g. MOSFETs, IGBTs or IGCTs), the devices are not allowed to turn off during current commutation. A good solution, is adding up twice the magnetization-time (rising, boosting and falling) of the auxiliary inductor plus the time of the resonant phase as defined in [2], in worst case condition. If the device would be turned off before the current decays to zero, the auxiliary inductor would cause an over-voltage condition at the device.

In Fig. 3 the SV given for each converter leg are depicted with the timing constrains for thyristor-based FQS and the ZSC. The resulting six-step voltages of the DAB and the resonant current $i_{\text {res }}$ of the ARCP are plotted below.

\section{A. Commutation Control}

The boost current is essential for a proper commutation. Whenever the current in the device that is about to be turned off is negative, and thus the anti-parallel diode is conducting, the current needs to be reversed for the commutation. In this operation mode, the current in the auxiliary branch must be higher than the load current to overcome losses during the 
TABLE II

Space Vectors (SV) and IGBT Triggering Signals (FQS) for One Converter

\begin{tabular}{cccc}
\hline \hline Time Sequence & $\mathrm{SV}$ & $\mathrm{FQS}$ & State \\
\hline$-T_{\text {discharge }}<t<0$ & 000000 & 000000 & 0 \\
$-T_{\mathrm{trg}}<t<+2 \cdot T_{\text {trg }}$ & 010101 & 010000 & $0-1$ \\
$0<t<T_{\mathrm{sw}} / 6$ & 100101 & 000000 & 1 \\
$T_{\mathrm{sw}} / 6-T_{\mathrm{trg}}<\mathrm{t}<T_{\mathrm{sw}} / 6+2 T_{\mathrm{trg}}$ & 100101 & 000100 & $1-2$ \\
$T_{\mathrm{sw}} / 6<t<2 T_{\mathrm{sw}} / 6$ & 101001 & 000000 & 2 \\
$2 T_{\mathrm{sw}} / 6-T_{\mathrm{trg}}<t<2 T_{\mathrm{sw}} / 6+2 T_{\mathrm{trg}}$ & 101001 & 100000 & $2-3$ \\
$2 T_{\mathrm{sw}} / 6<t<3 T_{\mathrm{sw}} / 6$ & 011001 & 000000 & 3 \\
$3 T_{\mathrm{sw}} / 6-T_{\mathrm{trg}}<t<3 T_{\mathrm{sw}} / 6+2 T_{\mathrm{trg}}$ & 011001 & 000001 & $3-4$ \\
$3 T_{\mathrm{sw}} / 6<t<4 T_{\mathrm{sw}} / 6$ & 011010 & 000000 & 4 \\
$4 T_{\mathrm{sw}} / 6-T_{\mathrm{trg}}<t<4 T_{\mathrm{sw}} / 6+2 T_{\mathrm{trg}}$ & 011010 & 001000 & $4-5$ \\
$4 T_{\mathrm{sw}} / 6<t<5 T_{\mathrm{sw}} / 6$ & 010110 & 000000 & 5 \\
$5 T_{\mathrm{sw}} / 6-T_{\mathrm{trg}}<t<5 T_{\mathrm{sw}} / 6+2 T_{\mathrm{trg}}$ & 010110 & 010000 & $5-6$ \\
$5 T_{\mathrm{sw}} / 6<t<6 T_{\mathrm{sw}} / 6$ & 100110 & 000000 & 6 \\
$6 T_{\mathrm{sw}} / 6-T_{\mathrm{trg}}<t<6 T_{\mathrm{sw}} / 6+2 T_{\mathrm{trg}}$ & 100110 & 000010 & $6-1$ \\
\hline \hline
\end{tabular}

commutation and to accomplish the commutation within a reasonable time. Here, two techniques are presented:

\section{1) Fixed Magnetization Time}

In terms of control effort, keeping the magnetization time constant is a straight forward approach. The magnetization must greater than the rising time and the minimal required boosting time. The FPGA is set up to trigger the FQS a certain time before the main switches transition. This time can either be kept constant overall operating points or adjusted by the controller according to system voltage and phase current amplitudes. Anyway changing the time according to the operation point of the converter can be challenging during operation.

Fig. 5(a) and (b) depicts the effect of changing system voltages on the resonant currents at a constant boost time.

Except for ease of control, this approach has the following drawbacks: for commutations at negative phase currents, constant magnetization time will increase the device stress during turn-off for the bottom main switches, as it adds the full resonant current to the load current, thus increasing the resulting current in the switch. This unnecessary current causes losses in all devices taking part in these commutations.

However, constant boost time has the natural capability of balancing the dc-link voltages. Whenever one of the two dclink capacitors connected in series is unbalanced, the auxiliary inductor is magnetized with a higher peak current. As shown in Fig. 5(c), an increased amount of energy is pushed into the corresponding capacitor.

\section{2) Constant Boost Current}

The drawbacks of a control based on equations and calculations for optimal boost time [10] can be overcome by dynamically terminating the boost sequence. This can be

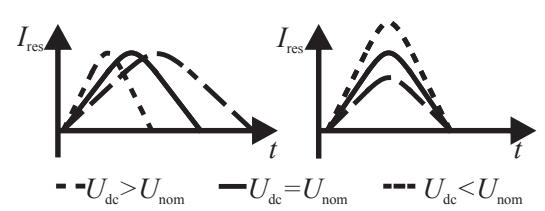

(a)

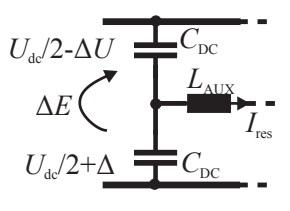

(c)
Fig. 5. Impact of dc-link voltage variation on constant boost current strategy (a) or constant boost time strategy (b) and increasing boost current during bottom to top transition for dc-balancing purposes (c).

achieved by monitoring the difference of resonant and load current $i_{\mathrm{RES}}-i_{\mathrm{L}}$ using an additional sensing. If this difference reaches a certain limit $I_{\lim }$, the FPGA will turn off the according main switch, stopping the inductor magnetization. This way, the influence of load current and voltage amplitude are rejected and the boost current is kept constant. The increased hardware effort is a clear drawback of this approach. Adjusting the turn-off time of the main switches according to the incoming signal is a small extension of the existing FPGA code.

\section{B. DC-Link Balancing Using Variable Boost Currents}

The ARCP circuit is connected to the midpoint of the dc-link, thus the DAB converter can be used in bipolar dc systems. For a system with unbalanced loads, the DAB ARCP can provide voltage balancing capabilities. The following subsections will discuss the natural and active dc-link balancing behavior of the ARCP as well as the control approach taken. A hysteresis controller enables active dc-link balancing for variable set and reset boundaries as shown in Fig. 9(c). Both, the upper and lower dc-link voltages are monitored and therefore the DSP is able to determine how to counteract the imbalanced voltages. Fig. 2 depicts the block 'turn off delay' which allows to extend the magnetization time of the auxiliary inductor. To balance the dc-links only the magnetization time for one component is extended. E.g. if the upper dc-link $U_{\mathrm{PRIM}, 1}$ is lower compared to the second one $U_{\text {PRIM }, 2}$, the boost time of the FQS2, FQS4 and FQS6 is extended. In case, the dc-link of $U_{\text {PRIM }, 2}$ is lower, the boost time of FQS1, FQS3 and FQS5 is adjusted. As a result the ARCP currents are asymmetrical and thus the power transferred between the dc-link capacitors is asymmetrical as well. The basic topology behind the ARCP is a push-pull converter. Based on this knowledge, it would be possible to define a transfer function with a disturbance coupling according the load current $i_{\mathrm{L}}$. Here, for simplicity, only a hysteresis controller is embedded. Whenever the voltage differs more than $20 \%$ from half the sum of the two measured dc-link voltages - nominal $U_{\mathrm{dc}} / 2$ - the hysteresis control is engaged until the difference of the virtual $U_{\mathrm{dc}} / 2$ is less than $2 \%$.

\section{Simulation of Proposed Control Techniques}

The simulations are performed in Matlab Simulink/PLECS and the VHDL code is integrated using the Xilinx System Generator. In Fig. 6, the results of the simulations are shown. At the beginning of the simulation, the zero-state chopper is activated until the resonant capacitors at the bottom switches are 

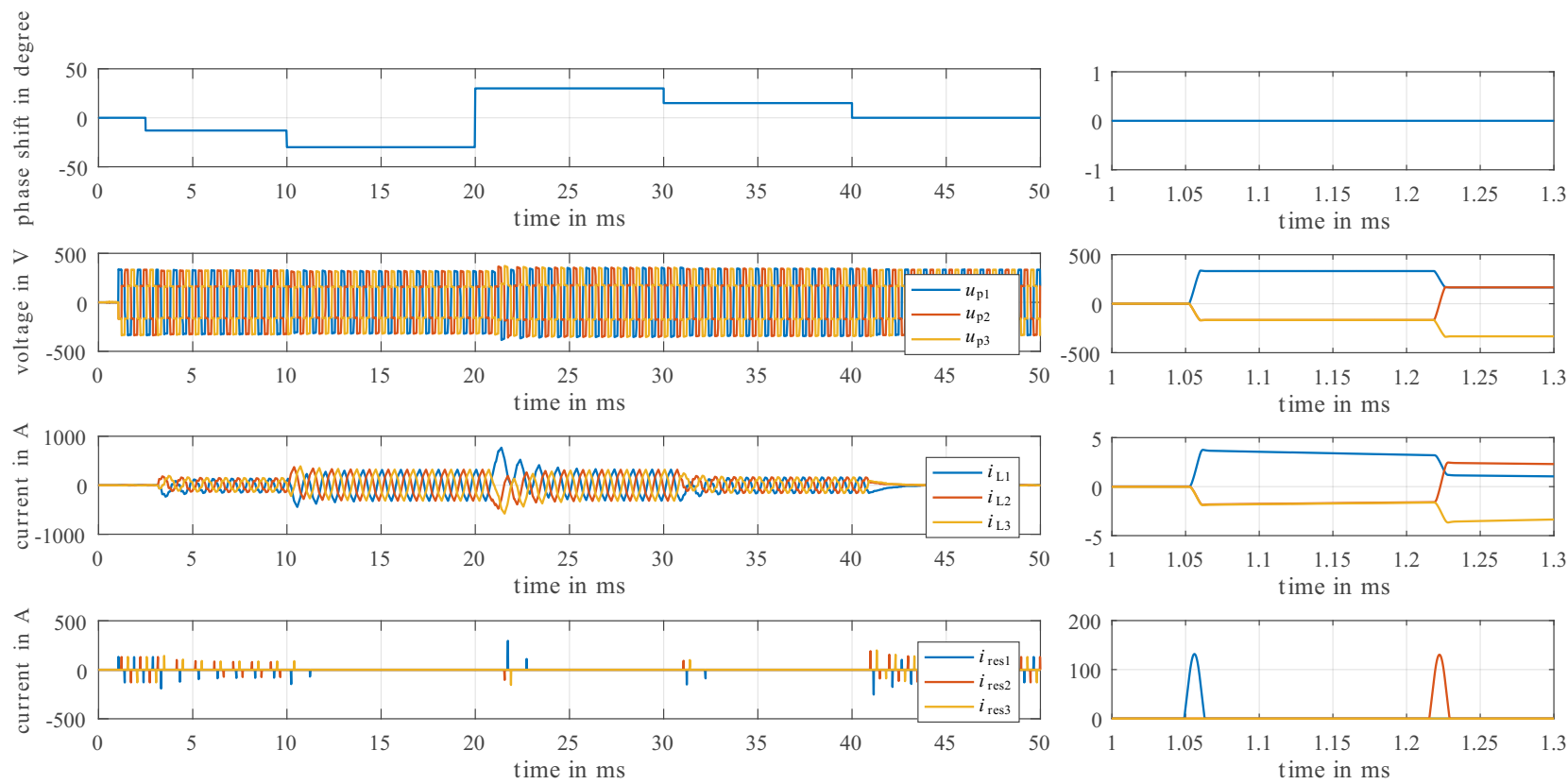

Fig. 6. Performed simulation of the FPGA code using the Xilinx System Generator.

completely discharged, equivalent to state 0 .

In the first plot of Fig. 6, the phase shift is given in degree. In the second plot, the phase voltages, phase currents and, finally the ARCP currents are depicted. On the right-hand side, a zoomed in of the start-up procedure is shown. Here, the converter is initiated, using the zero-state of the SVM. The transition of the first interval is supported with the ARCP branch, up to approximately $t=4 \mathrm{~ms}$.

The phase shift is increased afterwards. Due to transient effects which results in dc-voltage-offsets, the ARCP is activated only at some switching transitions since the phase current does not reach the specified minimum load current for natural soft switching.

At $t=10 \mathrm{~ms}$, the phase-angle is changed to its maximum and the converter is running at nominal load. Now the phase current is beyond the minimum load current for soft switching and the ARCP is left turned off. This operation area is named as natural soft-switching mode since no additional reactive power is needed to allow zero-voltage switching. The nominal power is reversed at $t=20 \mathrm{~ms}$. The very fast change of the load causes very high peak currents during the transition.

The current comparator equivalently reacts on this current and ensures soft-switching during this event. This could be avoided using ICC as proposed in [12]. However, using ICC additional timing constrains regarding minimum intermediate steps must be considered.

Afterwards, the power is decreased equally and the con-verter is running in forced soft-switching mode again.

\section{Small-Scale Laboratory Setup}

Here, the experimental setup of the small-scale dual-active bridge (SS SAB) is introduced. The utilized hardware is pictured in Fig. 7. The schematic is given in Fig. 8. The dc-link

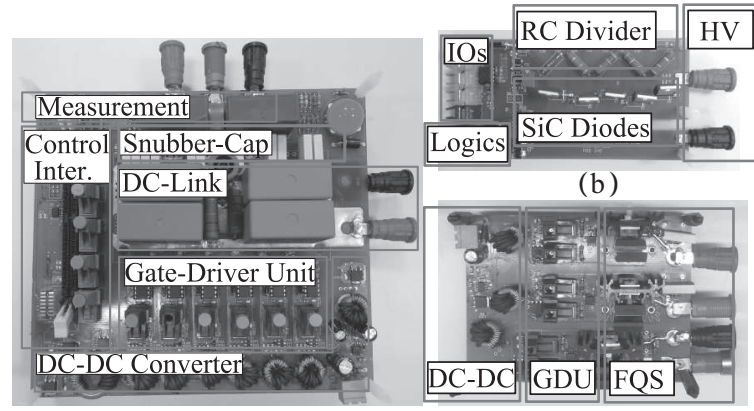

(a)

(c)

Fig. 7. Pictures of components in the experimental setup.

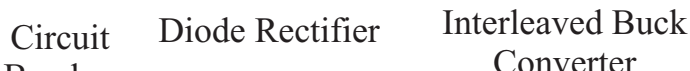

Breaker Converter

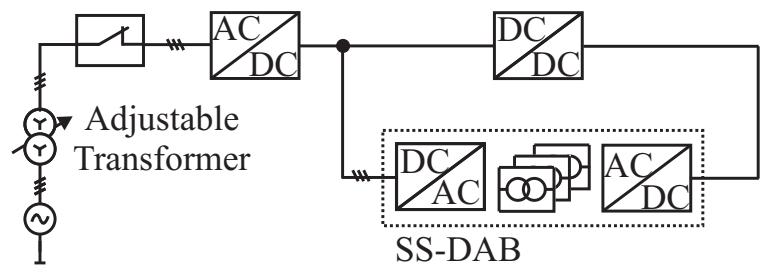

Fig. 8. Simplified schematic of the SS DAB laboratory setup.

is powered by an adjustable transformer with a diode rectifier to test the strategies under varying voltage conditions.

\section{A. Voltage-Source Converter}

The three-phase input and output converters as well as the buck converter are independent systems incorporating a fiber optic input/output interface (I/O), galvanically isolated GDUs, 

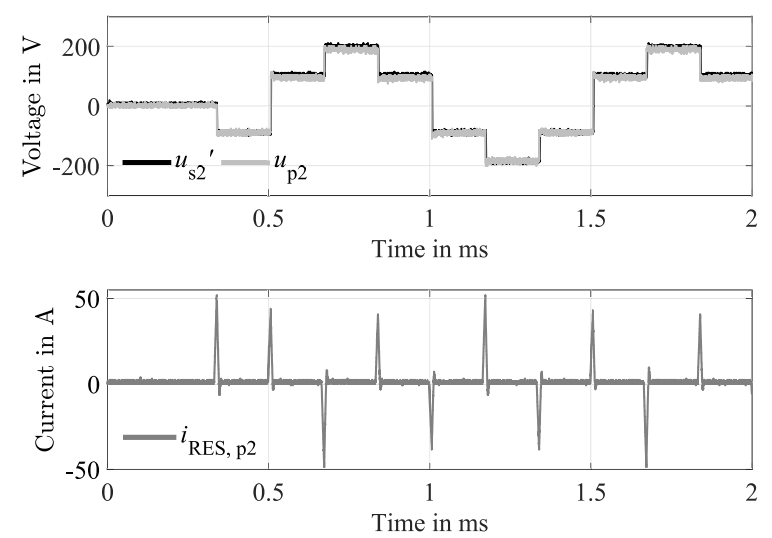

(a) Characteristic voltages of the DAB during start-up procedure for DAB$\mathrm{ARCP}$ operation and primary resonant currents of all phases.

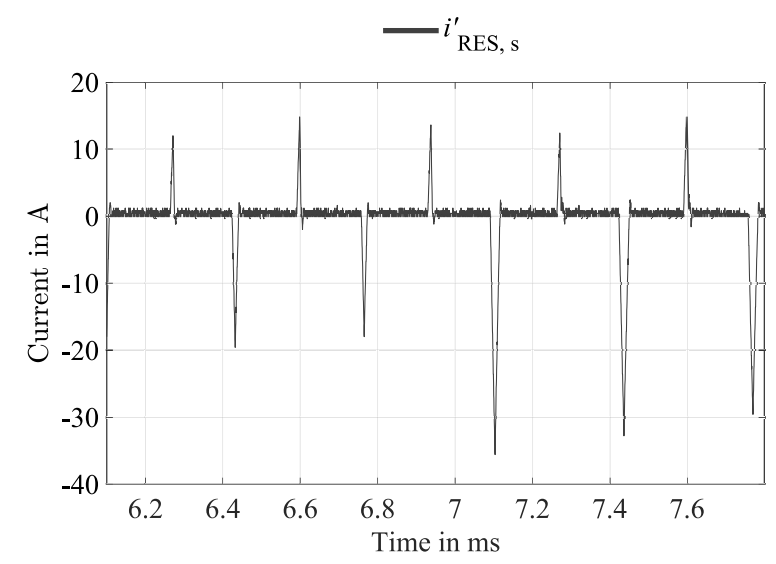

(c) Engaging active balancing of dc-links with hysteresis controller at $t=7 \mu \mathrm{s}$.

Fig. 9. Experimental results part 1.

an IGBT module and a heat sink. The IGBT modules are mounted under the PCB on a heat sink and not visible in Fig. 7(a). The converter is state-of-the-art topology with additional snubber capacitors mounted at the output side. If the converter is used as an independent system, a TI F28335 DSP can be plugged in and programmed via USB interface.

\section{B. Four-Quadrant Switches for ARCP}

In this work, both types of FQS based on thyristors and on IGBTs are used. Both topologies have advantages and disadvantages. From control perspectives, thyristors are beneficial since they turn off at a zero crossing. However, most common thyristors are not designed for higher switching frequencies, resulting in restrictions for $\mathrm{d} i / \mathrm{d} t$ and $\mathrm{d} U / \mathrm{d} t$ and during softstart sequences of the converter. Fig. 7(c) show a version with thyristors.

\section{Zero-Voltage Detection and Current Comparator}

The ZVD is necessary to prevent the main devices from switching while their snubber capacitor is still charged. IGCTs in general are sensitive to high $\mathrm{d} i / \mathrm{d} t$ slopes, as their structure
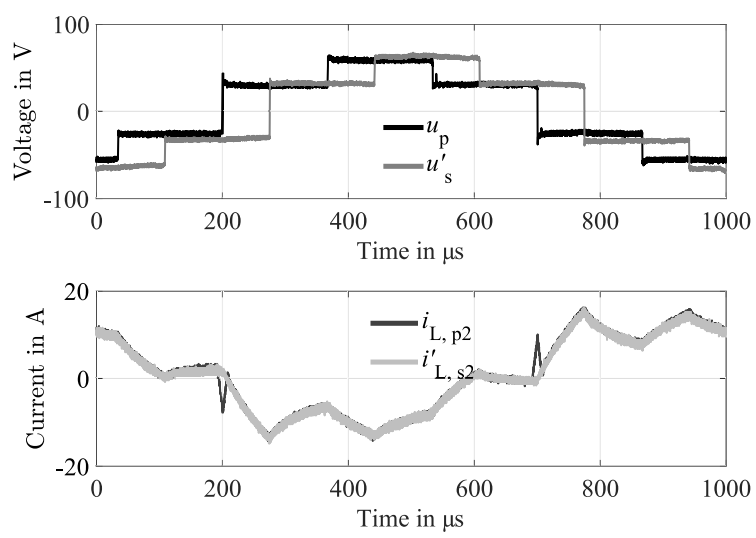

(b) Primary and secondary transformer voltages and phase currents with auxiliary current for primary side at $45^{\circ}$ phase shift. Boost currents visible in $I_{\mathrm{L}}$ at $t=200 \mu \mathrm{s}$ and $700 \mu \mathrm{s}$

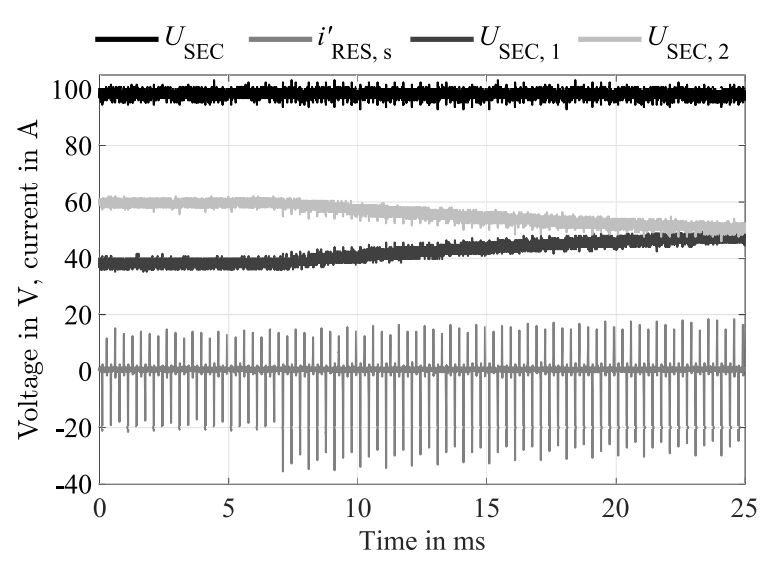

(d) Detailed view of $I_{\mathrm{ARCP}}$ in Fig. 9(c) around $t=7 \mu \mathrm{s}$.

can cause internal hot spots if a high current immediately after a turn on occurs [13]. Since the ZVD is designed for the $5 \mathrm{MW}, 5 \mathrm{kV}$ converter zero-voltage is defined as voltage below $20 \mathrm{~V}$. The ZVD is shown in Fig. 7(b).

In case of an early turn-on of the main switch, these additional devices would protect the main switches from shorting the snubbers, which is called a snubber dump. The detection PCB via the main switches in the main converter legs is directly mounted across the snubber capacitors. The basic logic is illustrated in Fig. 11. The PCB receives the gate turn-on signal from the FPGA. This signal is delayed and only passed on to the device once the voltage condition of less than $20 \mathrm{~V}$ is reached.

The circuit schematic of the ZVD is shown in Fig. 12. To block against the $5 \mathrm{kV}$, five $\mathrm{SiC}$ high-voltage (HV) diodes, each rated for $1.2 \mathrm{kV}$, are connected in series, with a typical forward voltage of $0.77 \mathrm{~V}$ at low currents, as indicated in Fig. 12. Their capacitance is stated to be $59 \mathrm{pF}$ at $800 \mathrm{~V}$. They are connected in series and terminated to ground with a Zener diode $U_{\mathrm{Z}}=20 \mathrm{~V}$. The voltage across the $\mathrm{SiC}$ diodes is balanced with an ohmic divider. Each resistor is rated for $470 \mathrm{k} \Omega$. In order to maintain a predictable behavior, to reject parasitic elements and to provide a stable delay over a wide range of operating points, a constant 


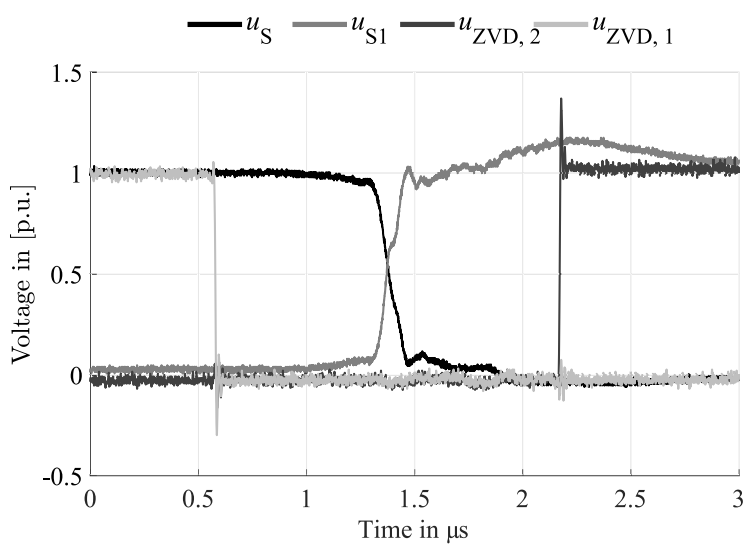

(a) Detailed voltages during a resonant commutation and voltage input of the fiber optic receiver at the main switch GDU generated by the ZVD.

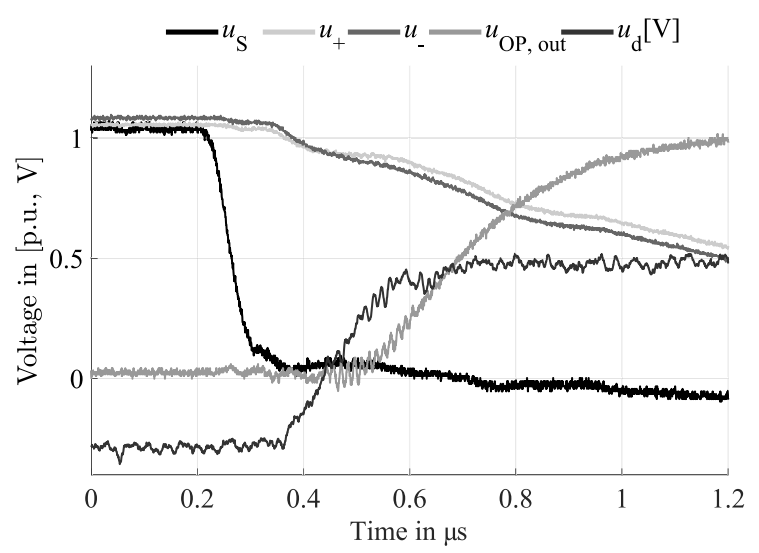

(c) Detailed probe signals of ZVD during test at $80 \mathrm{~V}$.

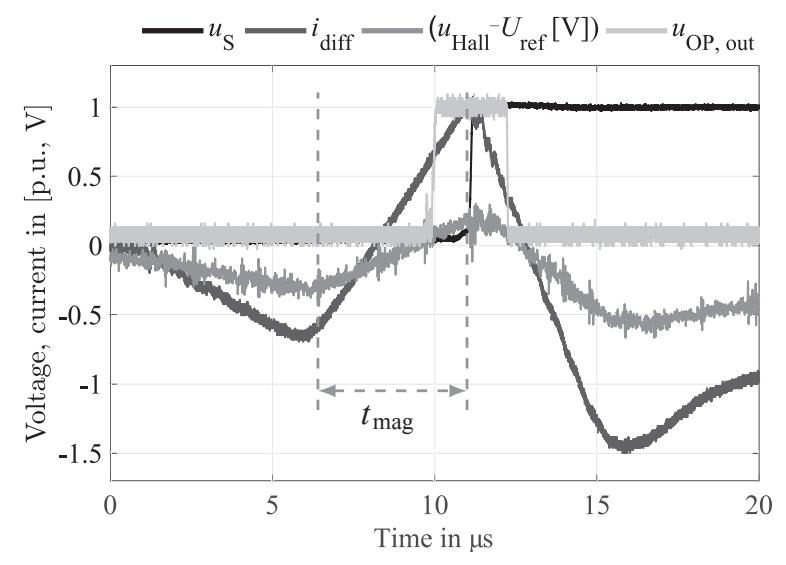

(e) Constant boost current at negative load current condition.

Fig. 10. Experimental results part 2. measurements in (a) to (e) are in p.u.

current source supports the detection circuit. This current source is constantly feeding approximately $30 \mathrm{~mA}$ into the circuit. Depending on the switching state of the main switch, this current will flow through the Zener diode or into the switching path. Thus, the provided energy keeps the voltage across the Zener diode high until the current changes paths into the HV

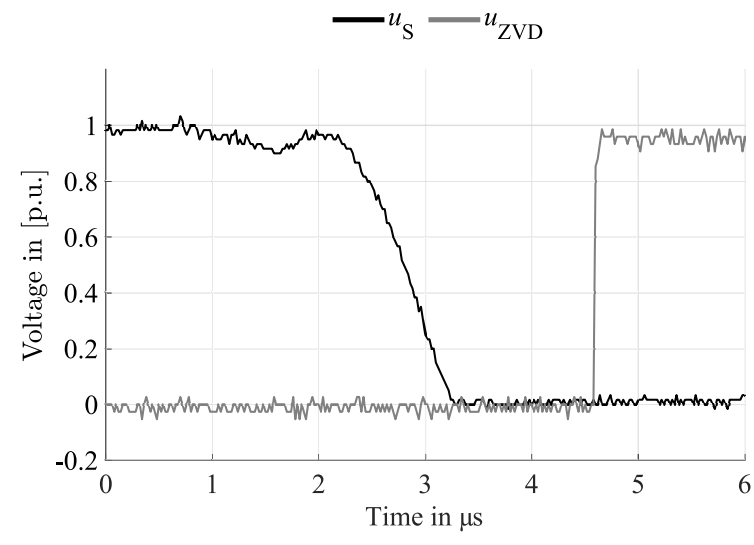

(b) Voltage of main switch and delay of ZVD during a test at $1.2 \mathrm{kV}$.

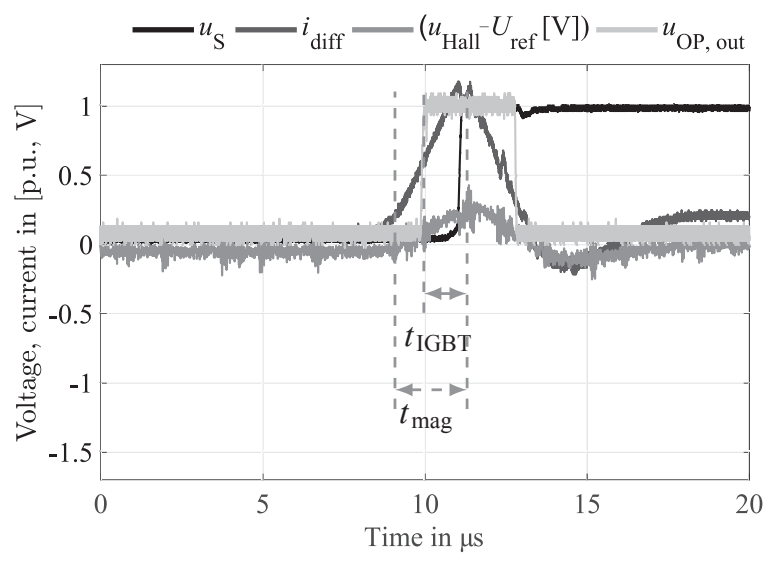

(d) Constant boost current control at no load current condition.

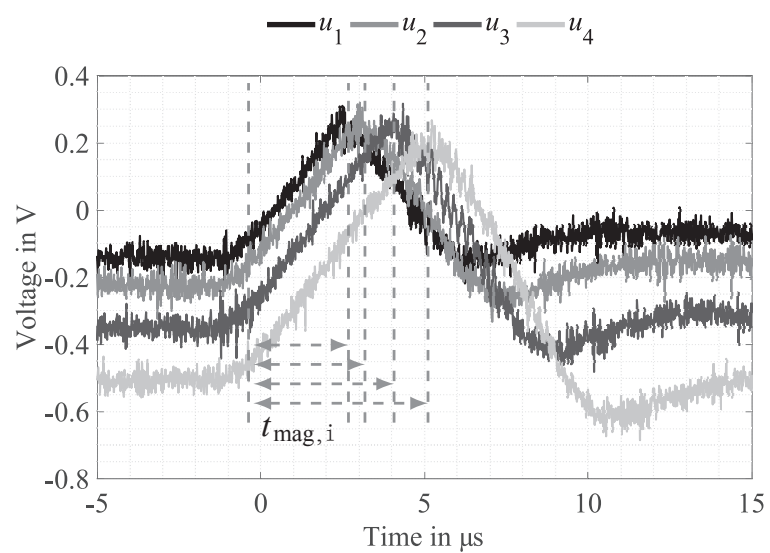

(f) $U_{\mathrm{i}}=U_{\text {Hall }}-U_{\text {ref }}$ on current comparator at various operating points resulting in different magnetization times and constant current amplitudes.

diodes. Compared to a voltage source, no additional capacitors are used and the parasitic capacitance of the Zener diode is not increased. An adjustable voltage divider provides a reference voltage $U_{+}$for the comparator as indicated in Fig. 12. For low voltages across the switch or a short between $U_{\mathrm{S}}$ and ground, the current source will feed current into the switching path 


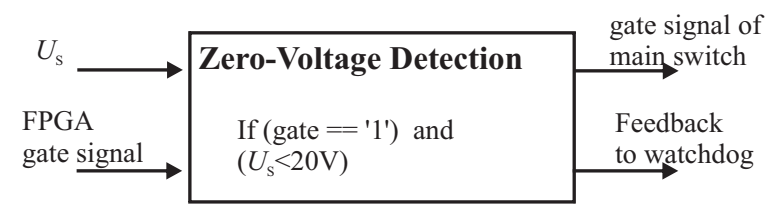

Fig. 11. Logic of zero-voltage detection.

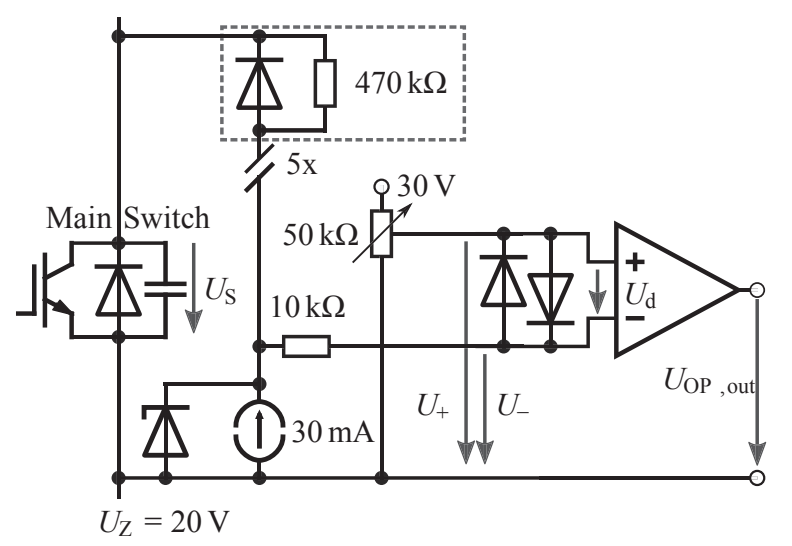

Fig. 12. Schematic of the analogue part on the zero-voltage detection PCB.

and thus pull $U$ down and forward bias the protection diode in front of the comparator. Therefore, $U_{\mathrm{d}}$ is positive. Since the operational amplifier acts as a comparator, the output resembles a logical high for $U_{\mathrm{d}} \geq 0 \mathrm{~V}$. In the opposite case, at a voltage greater than $20 \mathrm{~V}$ across the switch, the Zener diode will pull up $U$. By adjusting the reference voltage to be slightly below the Zener voltage, the delay produced by the ZVD can be minimized. Although, a safety margin should be kept in order to provide more stability. The output $U_{\mathrm{OP}, \text { out }}$ is linked to the gate signal from the FPGA on a logic AND gate to generate the final main switch gate signal.

Another critical feature of a ZVD is its delay chain. In order to turn on the main switch as long as zero-voltage conditions are present, the ZVD should not withhold the gate signal too long. During a resonant commutation, an interval of a few micro seconds exists where the main switch is turned on under zero-voltage conditions. Delays inside the ZVD are caused by the parasitic charging, the lag of the comparator and the logic "AND" IC. The first two can be observed in Fig. 10(c). For this experiment, the reference is set close to the Zener voltage, so the delay between $U_{\mathrm{SW}}$ being smaller than $20 \mathrm{~V}$ is as short as possible. Here, it is about $100 \mathrm{~ns}$. The time between $U_{\mathrm{d}}>0 \mathrm{~V}$ and the output of the comparator reaching high is about $0.5 \mu \mathrm{s}$.

\section{Current Comparator}

The comparator is needed to compare the current in the auxiliary branch $I_{\text {RES }}$ to the load current $I_{\mathrm{L}}$. Whenever $I_{\text {RES }}$ is higher than the $I_{\mathrm{L}}$, it can be ensured that boost current is flowing into the diode during commutation. The phase to resonant current comparator is logic high whenever the current $I_{\mathrm{DIFF}}=I_{\mathrm{RES}}-I_{\mathrm{L}}$ is higher than a predefined limit. Since the boost current is only present during a commutation, it takes an unreasonable amount of effort to

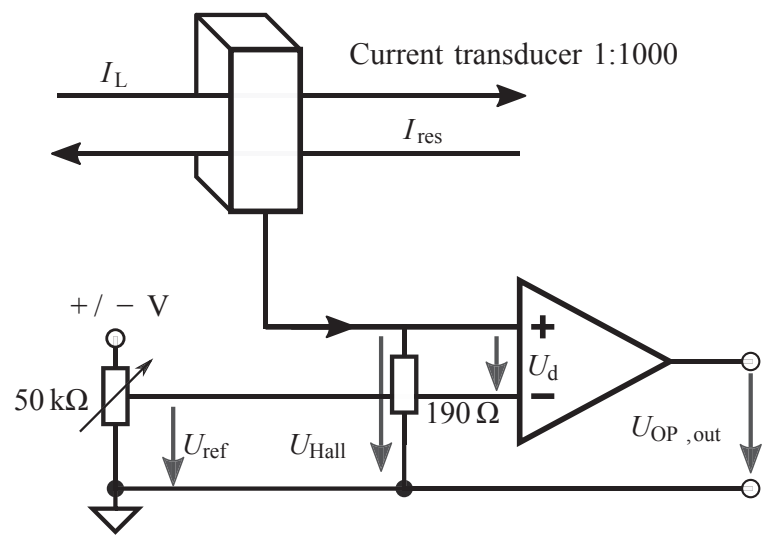

Fig. 13. Schematic of the analogue part on the current comparator PCB.

measure it directly with the DSP. Interrupt times can make it very difficult to measure with the needed speed and/or precision. It is possible to obtain the amplitude of the resonant current by measuring the phase and resonant currents and subtracting them from each other. Typical boost times are well below $15 \mu \mathrm{s}$. An implementation of a detection algorithm in software would delay the result for too long and the current would increase far over the desired limits. Since the FPGA is much faster compared to the DSP interrupt sequence, the approach here is to embed the function in an analogous platform.

The basic principle can be described as depicted in Fig. 4 on the right side and Fig. 13 respectively. Hall-based current transducers transform the auxiliary current and the load current in such a way that the fields within the sensor compensate. With a ratio of 1:1000, the current is injected into a highprecision measuring resistors causing a voltage drop equivalent to the boost current. The resistor is connected to an operational amplifier with a high differential voltage capability. This voltage is then compared to a reference created by a variable voltage divider. Whenever the comparator detects a voltage $U_{\text {Hall }}$, representing the boost current $I_{\text {boost }}=I_{\text {res }}-I_{\mathrm{L}}$, higher than the reference voltage, the main switch is turned off. This initiates the resonant commutation as stated in Fig. 5. The amplitude of the boost current can be adjusted by setting the reference voltage to the desired value. The reference voltage represents the minimum current $\left|I_{\min }\right|$ which is needed to allow a commutation within a specified time as explained in [8]. As shown in Fig. 2, the dead time must be compensated since the main switch turn-off is delayed compared to a fixed boost time control, effecting the turn on of its complementary switch. Additionally, the delays introduced by this PCB have to be taken into account. Again, a chain of delays caused by the operational amplifier, fiber optic I/O, FPGA computation time and the turn off time of the main switch $t_{\mathrm{IGBT}}$ can be observed. For this application, the delay of the main switch is the major contributor to the overall delay. Note that an increased delay causes an extended magnetization time and, therefore, a tremendously increased boost current.

\section{EXPERIMENTAL RESULTS}

In Fig. 9 and Fig. 10, the experimental results are summarized. 


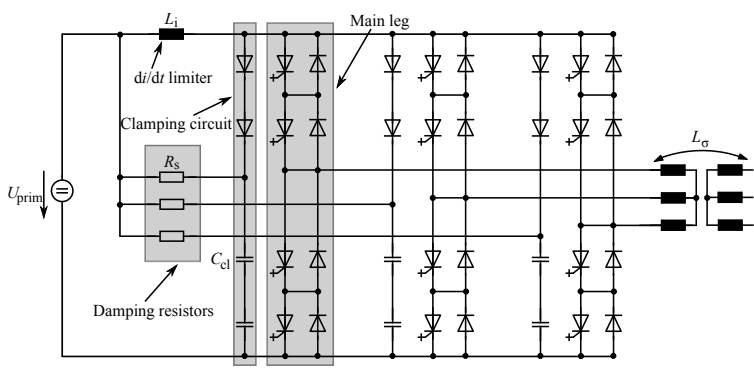

Fig. 14. Conventional snubbers for IGCT-based converters.

TABLE III

Cost Comparison of Conventional IGCT SnubBers Circuit and ARCP SNUBBERS

\begin{tabular}{|c|c|c|c|}
\hline & Component & No. of Dev. & Est. Price \\
\hline & $\mathrm{d} i / \mathrm{d} t$ limiter & 2 & $12.5 \mathrm{t} €$ \\
\hline Conventional & Clamping diode & 12 & $8.4 \mathrm{t} €$ \\
\hline \multirow[t]{5}{*}{ Snubber } & Clamping capacitor & 12 & $4.8 \mathrm{t} €$ \\
\hline & Damping resistor & 6 & $3.6 \mathrm{t} €$ \\
\hline & Snubber capacitor & 48 & $1.5 \mathrm{t} €$ \\
\hline & Auxiliary inductor & 2 & $1.6 \mathrm{t€}$ \\
\hline & Auxiliary switch (IGBT) & 12 & $15.6 \mathrm{t} €$ \\
\hline ARCP & Radiators for IGBTs & 12 & $1.1 \mathrm{t} €$ \\
\hline \multirow[t]{5}{*}{ Snubber } & GDUs IGBTs & 12 & $0.96 \mathrm{t} €$ \\
\hline & ZVD PCB & 12 & $0.5 \mathrm{t} €$ \\
\hline & ZCD PCB & 12 & $0.8 \mathrm{t} €$ \\
\hline & Hall-based sensors & 12 & $1.2 \mathrm{t€}$ \\
\hline & Total amount & & $-6.0 \mathrm{t} €$ \\
\hline
\end{tabular}

In Fig. 9(a) and (b), the main operation of the converter is shown. Beginning with the start of the converter, the steadystate voltages and the resonant currents are shown in Fig. 9(a). Whenever a phase-shift is applied, the load current and the resonant current are added up as it can be observed at $t$ $=200 \mu \mathrm{s}$ and $t=700 \mu \mathrm{s}$ in Fig. 9(b). In Fig. 10(a), a detailed voltage commutation in a converter leg and the ZVD signals are shown. Fig. 10(b) and (c) show the ZVD. In Fig. 10(b) a high-voltage test has been conducted and the delay of about $1 \mu$ s can be achieved over a wide voltage range. To learn more about the total delay, detailed measurements are taken at lower voltages and plotted in Fig. 10(c). Measurements taken during tests of the constant boost current mode are depicted in Fig. 10(d)-(f).

These measurements show the successful application of the introduced control techniques on a small scale test bench and validate the simulations used to design the VHDL structures. Future measurements on the full scale DAB will provide insights on efficiency improvements achieved by these techniques.

Conventional systems using IGCTs need $R C D$ snubbers and $\mathrm{d} i / \mathrm{d} t$ limiter to fulfill the SOA of the IGCT devices as shown in Fig. 14. In the high-power setup, the applied IGCTs 5SHY35L4510 have $\mathrm{d} i / \mathrm{d} t$ limitations of $800 \mathrm{~A} / \mu \mathrm{s}$. The modification from a classical clamping to an ARCP system will affect the number of used components. However, the conventional snubber systems are not needed anymore. In TABLE III a cost comparison between conventional snubber and usage of an ARCP with IGBTs as auxiliary switches is given. The table considers the investment costs of the three-phase high-power DAB converter. For conventional snubbers an investment of $29.3 \mathrm{t} €$ for the high-power setup is needed. The ARCP circuit requires an investment of $23.3 \mathrm{t} €$. Embedding the ARCP would result in 6.0 t€ savings, plus the efficiency improvements which will cumulate more savings over time. A big price difference can be seen between the $\mathrm{d} i / \mathrm{d} t$ limiter, the auxiliary inductors and the switches. In conventional clamping circuit the $\mathrm{d} i / \mathrm{d} t$ limiter must be rated for the total dc-link current in the existing high-power setup it is water-cooled resulting in an expensive design. The auxiliary resonant inductor is only rated for the rms current during the resonant phase.

\section{Conclusion and Outlook}

The auxiliary-resonant commutated pole seems to be an interesting technique for reducing the switching losses of a high-power $5 \mathrm{MW}$ dc-dc converter based on IGCTs. The basic control is explained using space vectors and the given timing constrains regarding the ARCP branch are introduced. As a four-quadrant switch either line-commutated or forced-commutated devices can be used. The modulation is verified in a small-scale setup with FPGA/DSP based controller. The two presented boosting strategies, constant magnetization time and constant boost current, are tested in the experimental setup and results are shown. For the ARCP concept a high-speed ZVD is design and explained with a delay of approximately $1.5 \mu \mathrm{s}$. To compensate the delay time analog tuning is necessary. Finally, the use of an ARCP to balance the dc-link capacitors is introduced, allowing the DAB converter to operate in bipolar systems with unbalanced loads. The small-scale dual-active bridge proves the proper functionality of the control techniques. The boosting strategies can be applied directly to the $5 \mathrm{MW}$ converter. In the near future the developed control will be implemented in a high-power dc-dc converter and tested to show improvements in IGCT switching behavior and an increase in efficiency.

\section{AcKNOWLEDGMENT}

The authors would like to thank Infineon Technology AG for donating various components.

\section{REFERENCES}

[1] R.W. De Doncker, D. M. Divan, and M. H. Kheraluwala, "A threephase soft-switched high-power-density DC/DC converter for highpower applica-tions, "IEEE Trans. Ind. Appl., vol. 27, no. 1, pp. 6373, Jan./Feb. 1991.

[2] R.W. De Doncker and J.P. Lyons, "The auxiliary resonant com- 
mutated pole converter," in Industry Applications Society Annual Meeting, 1990

[3] Lutz, Schangenotto, Scheuermann, De Doncker, Semiconductor Power Devices, Physics Characteristics, Reliability, Springer-Verlag Berlin Hei-delberg, 2011,DOI 10.1007/978-3-642-11125-9.

[4] W. Dong, J. -Y. Choi, F. C. Lee, D. Boroyevich, and J. Lai, "Comprehensive evaluation of auxiliary resonant commutated pole inverter for electric vehicle applications," in 2001 IEEE 32nd Annual Power Electronics Specialists Conference (IEEE Cat. No.01CH37230).

[5] S. Karys, "Selection of resonant circuit elements for the ARCP inverter," in 2009 10th International Conference on Electrical Power Quality and Utilization, 2009.

[6] H. W. van der Broeck, H. C. Skudelny, and G. V. Stanke, "Analysis and realization of a pulsewidth modulator based on voltage - space vectors," IEEE Transanctions on Industry Applications, 1988.

[7] S. Bernet, R. Teichmann, J. Weber, and P. K. Steimer, "Evaluation of a high power ARCP voltage source inverter with IGCT," in Industry Applications Conference, 1999. Thirty-Fourth IAS Annual Meeting. Conference Record of the 1999 IEEE.

[8] R. Lenke, "A contribution to the design of isolated DC-DC converters utility applications," Dissertation RWTH Aachen University, 2010.

[9] K. Ma, D. Xu, T. Zhang, and S. Igarashi, "The evaluation of control strategies for auxiliary resonant commutated pole inverter, " in 2009 IEEE Energy Conversion Congress and Exposition.

[10] J. Voss, B. Bagaber, R. W. De Doncker, "Full soft-switching capability of the dual-active bridge by using the auxiliary-resonant commutatedpole technique," in IEEE 8th International Symposium on Power Electronics for Distributed Generation Systems, PEDG, Florianopolis, Brazil, April 2017.

[11] J. Voss, J. Henn, and Rik W. De Doncker, "Control techniques of the auxiliary-resonant commutated pole in the dual-active bridge, " in The 12th IEEE International Conference on Power Electronics and Drive Systems, 2017, Honolulu, Hawaii, USA, 12th to 15th December 2017.

[12] S. Engel, N. Soltau, H. Stagge, and R. W. De Doncker, "Improved instantaneous current control for high-power three-phase dual-active bridge DCDC converters," IEEE Transaction on Power Electronics, vol. 29, no. 8, August 2014.

[13] ABB Switzerland Ltd, Applying IGCTs, Application Note 5SYA 20324, 16 June 2016

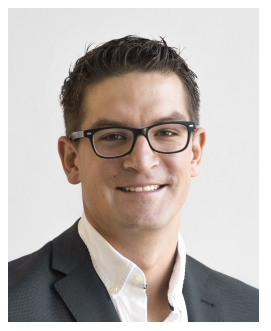

Johannes Voss joined the Institute for Power Generation and Storage Systems (PGS), E.ON Energy Research Center, RWTH Aachen University, Aachen, Germany, in 2012. In 2011, he received his Diploma in electrical engineering from RWTH Aachen University. In 2016 he participated as an exchange student for 8 months the NSF Center for GRid-connected Advanced Power Electronic Systems (GRAPES) at the University of Arkansas. His research interests include the field of power

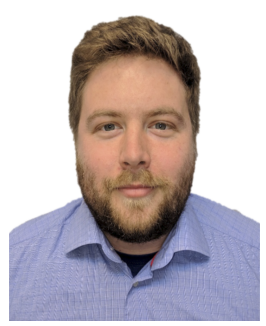

Jochen Henn joined the Institute for Power Electronics and Electrical Drives (ISEA) at RWTH Aachen University, Aachen Germany in 2017. In 2017 he received his M. sc. in electrical Engineering from RWTH Aachen University. His research interests include the field of power electronics.

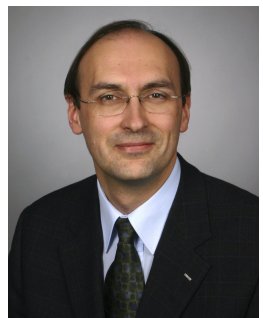

Rik W. De Doncker received the Ph.D. degree in electrical engineering from the Katholieke Universiteit Leuven, Leuven, Belgium, in 1986. In 1987, he was appointed as a Visiting Associate Professor at the University of Wisconsin, Madison. After a short stay as an Adjunct Researcher with Interuniversity Microelectronics Centre, Leuven, he joined, in 1989, the Corporate Research and Development Center, General Electric Company, Schenectady, NY. In 1994, he joined Silicon Power Corporation, a former division of General Electric Inc., as the Vice President of Technology. In 1996, he became a Professor at RWTH Aachen University, Aachen, Germany, where he currently leads the Institute for Power Electronics and Electrical Drives. Since 2006, he has been the Director of the E.ON Energy Research Center, RWTH Aachen University.

Dr. De Doncker was the President of the IEEE Power Electronics Society (PELS) in 2005 and 2006. He was the founding Chairman of the German IEEE Industry Applications Society PELS Joint Chapter. In 2002, he was the recipient of the IEEE IAS Outstanding Achievement Award. In 2008, he received the IEEE PES Nari Hingorani Custom Power Award. In 2009, he led a VDE/ETG Task Force on Electric Vehicles. In 2010, he received an honorary Ph.D. from TU Riga, Latvia. In 2013, he received the IEEE William E. Newell Power Electronics Award.

electronics. 